\title{
Network analyses reveal the role of large snakes in connecting feeding guilds in a species-rich Amazonian snake community
}

\author{
Daniela Coelho ${ }^{1}$, Marcio Martins ${ }^{2}$, and Paulo Guimarães Jr. ${ }^{2}$ \\ ${ }^{1}$ Universidade de São Paulo \\ ${ }^{2}$ Universidade de Sao Paulo
}

January 21, 2021

\begin{abstract}
In ecological communities, interactions between consumers and resources lead to the emergence of ecological networks and a fundamental problem to solve is to understand which factors shape network structure. Empirical and theoretical studies on ecological networks suggest predator body size is a key factor structuring patterns of interaction. Because larger predators consume a wider resource range, including the prey consumed by smaller predators, we hypothesized that variation in body size favors the rise of nestedness. In contrast, if resource consumption requires specific adaptations, predators are expected to consume distinct sets of resources, thus favouring modularity. We investigate these predictions by characterising the trophic network of a species-rich Amazonian snake community (62 species). Our results revealed an intricate network pattern resulting from larger species feeding on higher diversity of prey, promoting nestedness, and specific lifestyles feeding on distinct resources, promoting modularity. Species removal simulations indicated that the nested structure is favored mainly by the presence of five species of the family Boidae, which because of their body size and generalist lifestyles connect modules in the network. Our study highlights the particular ways traits affect the structure of interactions among consumers and resources at the community level.
\end{abstract}

\section{Introduction}

Interactions between species are a key component for understanding biodiversity (Abram 1987). In fact, individuals of all species rely upon ecological interactions to obtain food, to breed, or to protect against natural enemies (Thompson 2005). Ecological interactions form networks that connect populations of different species in a locality (Bascompte and Jordano 2013). The organisation of these networks may have important conservation implications, potentially affecting the robustness of ecological systems to species loss (Schmitz and Beckerman 2007). In this context, it is essential to understand how factors that influence the interactions between individuals affect the structure of networks at the level of ecological communities.

The structure of several ecological networks generally deviates from what is expected for networks in which individuals interact randomly, i.e., the interaction is proportional to the product of species abundances (Krishna et al. 2008). These deviations from expected network structure suggest that factors such as the characteristics of interacting individuals and environmental conditions influence the structural patterns of ecological networks at the community level. Among the traits that may affect network structure is body size, which is directly associated with the ability of individuals to consume resources (Stouffer et al. 2011). If the larger the predator individual, the greater its ability to kill larger prey we should expect, at the species level, larger average body sizes to be correlated with a larger number of resource types consumed by predator species (Sinclair et al. 2003). Furthermore, if only body size were influencing the capacity to consume a wider range of resources, it is expected that the diet of the smaller predator species will be a subset of the items of the larger predator species's diet, leading to nested ecological networks (Sinclair et al. 2003, Woodward et al. 2005, Stouffer et al. 2011). 
On the other hand, food resources are not homogeneously distributed in environments and the degree of specialisation in the consumption of distinct sets of prey may require distinct adaptations (Schoener 1968, Covich and McDowell 1996). For instance, the Anolis lizards of South Bimini islands divide habitat and the food resources according to lizard average size classes, in which larger lizard species usually eat larger food items than smaller lizard species (see Schoener 1968). Thus, we can expect that due to the restrictions related to prey handling, prey detection, or nutritional yield, larger predators are predisposed to disregard smaller prey (Mittelbach 1981, Arnold 1993, Arim et al. 2010). In this sense, deviations from the perfectly nested pattern are expected, enabling the formation of semi-isolated groups (modules) in the network. Networks with a modular structure have stimulated much interest due to its possible evolutionary and ecological consequences (Ings et al. 2009). For instance, modules may represent coevolutionary units (Thompson 2005) and increase the stability of ecological networks, thus providing a potential mechanism through which complexity arise and persist in ecological communities (Krause et al. 2003, Ings et al. 2009).

Here, we explore the trophic network organisation of a community Amazonian snakes. Snakes have been used as a model system in studies on the effect of ecological interactions on diversity (Martins et al. 2001, Colston et al. 2010, Alencar et al. 2013, Bellini et al. 2015, Klaczko et al. 2016, Alencar et al. 2017). These studies explore how ecological traits, interspecific interactions, habitat use, and evolutionary history influence the current trophic interactions of different species of snakes. As snakes evolved morphological and behavioral adaptations to kill and ingest their prey whole (Greene 1983), traits related to different dietary habits of species make snakes a model study system to understand how trophic interactions organise community structure (Shine and Bonnet 2000).

Motivated by understanding the trophic organisation pattern of snake communities, we here use as a model a rich and well-studied community of Amazonian snakes (Martins and Oliveira 1998). We characterised the structure of the interaction network between snakes and their food resources. We expected that if only snake body size were shaping network patterns at the species level, the structure would be nested; on the other hand, if specialization in resource consumption were driving patterns of resource use across snakes, modularity would be expected. We then evaluated the role of different snake species and the effect of snakes' habitat use (referred to here as a lifestyle) in shaping the network structure.

\section{Methods}

\section{The network structure of interactions between snakes and their food resources}

We analysed the snake diet derived from a long-term study carried out in a Central Amazonia site on the natural history of forest snakes (Martins and Oliveira 1998). We described the resource use by snakes as an interaction matrix $\mathbf{A}$ in which if a snake $i$ feeds on a given resource $j$ and zero otherwise. The matrix Adefines a bipartite network in which snakes and resource types depict two distinct set of nodes and links describe interactions between snake species and food resources. Our food resources are not described at species level but at coarse categories such as small mammals, medium mammals and big mammals. Similar approach led to insights in the study of food webs (Cohen 1977) and individual-based networks (Araújo et al. 2008). In fact, there is no intrinsically correct level of description when characterizing an ecological network (Guimarães 2020). We opted for these coarse categories because they are in agreement with (i) the evidence that snakes are specialized in broad categories of resources, e.g., serpentiform organisms that include snakes, amphisbaenians, and caecilians (see Martins and Oliveira 1998); (ii) the level of detail available from the diet analyses of snakes. Having said that, to verify if our level of network description affects our analyses we performed a set of sensitivity analyses (details below).

We used four metrics to characterise the structure of the interactions network analysed: (i) degree distribution, which is the description on how the number of food resources a given snake can feed on (the degree) varies across snake species; (ii) connectance $(C)$, which is the proportion of all possible interactions actually recorded in the network. Connectance values range from 0 (non-connected network) to 1 (maximum connectance); (iii) modularity ( $M$ ), a measure of the extent to which the network is formed by groups (modules) of snake species in which snake within a module overlap in much of their resources, whereas snakes 
in different modules show no or weak resource use overlap; and (iv) nestedness $(N)$, which consists of an interaction pattern in which the specialists interact with sets of resources with which the generalists also interact. Detailed descriptions of the metrics are available in the Supplementary material Appendix 1.

We used $Q_{B}$ metric, defined by Barber (2007), to characterise modularity, with values ranging from 0 (nonmodular network) to 1 (completely modular). A simulated annealing algorithm (Guimerà \& Amaral 2005) was used to optimise the $Q_{B}$ value. Modularity analyses were performed using the Modular program (Marquitti et al. 2014). All the above and the following analyses were performed using $\mathrm{R}$ version 3.5.1 (R Core Team 2018), with the exception of modularity. We performed a set of sensitivity analyses to verify if our results are dependent on our approach to compute modularity (Supplementary material Appendix 1).

The NODF metric was used to characterise the nestedness degree (Almeida-Neto et al. 2008) and its values ranges from 0 (non-nested network) to 100 (perfect nestedness). The degree of nestedness and modularity were then compared with a theoretical benchmark provided by the null model 2 of Bascompte et al. (2003) (see detailed description in Supplementary material Appendix 1). We generated 1000 null model matrices to estimate nestedness and modularity. If a network shows a degree of nestedness or modularity larger than expected by the null model 2, then there is evidence of ecological or evolutionary processes acting on the network organisation that goes beyond those shaping the degree of specialisation of the snake species (e.g, Bascompte et al. 2003).

\section{The role of snake species in network structure}

If the network of interactions analysed follows the organisation pattern structured by body mass (i.e. presenting higher nestedness than expected by the null model 2), we hypothesized snake average body mass to be positively correlated with the number of resources consumed by the snake species. To explore this prediction, we investigate the association between average body mass and the role of species in the network structure. We recorded the estimates of the average body mass of each snake species in our network (data available in Feldman et al. 2016). Average body mass was log-transformed prior to analysis (Supplementary material Appendix 2 Table A1).

In order to understand the individual contribution of each species of snake to nestedness, we used a jackknife resampling approach in which we removed a snake species and recomputing the degree of nestedness. We repeated the procedure for all snake species in the network and then we computed a change in nestedness: $\Delta N_{\imath}=N-N_{\imath}$, in which $N$ is the degree of nestedness of the complete network and $N_{i}$ is the degree of nestedness after the removal of a snake species $i$. If body size is shaping the contribution to nestedness, we should expect that the $\Delta N_{\imath}$ will assume increasingly positive values as larger snakes are removed from the network, indicating that nestedness is higher in the presence of these larger snake species.

\section{The relationship between lifestyles and network structure}

Because dietary specialisation in snakes can be related to habitat occupation (see Martins et al. 2002, Alencar et al. 2017), we expect snake lifestyles to affect the degree of dietary specialisation (e.g., an aquatic snake would rely upon aquatic prey). If this is true, the distribution of lifestyles in the different modules will not be random. We evaluated this prediction using two analyses. First, we analysed the frequency of snake lifestyles in different modules. We estimated the probability of the observed number of species of a given lifestyle in a given module be reproduced by randomly assigning species across modules, but preserving the number of snake species in each lifestyle and the number of snake species in each module $(\mathrm{n}=1000$ randomisations). Then, we analysed the dissimilarity on lifestyles between modules. To do so, we used the Bray-Curtis index, available in vegan package in R (Oksanen et al. 2018) (see detailed description in Supplementary material Appendix 1). Dissimilarity between a pair of modules range from 0 (modules are identical in the composition of lifestyles) to 1 (no lifestyle occurs in both modules).

\section{Sensitivity analyses}

Sampling effects may affect the description of network patterns. Therefore, we performed a sensitivity analysis to explore how robust is the description of network patterns to changes in our dataset. We add 
information to the use of resources by snakes by using data from other Amazonian regions, based on evidence that there is no significant intraspecific variation on the snake's diet across different localities in Amazonia (Martins and Oliveira, 1998; Supplementary material Appendix 1 and Appendix 2 Table A1).

Snake diet often include food resources that are main consumed and resources that are only eventually consumed. We performed a sensitivity analysis to check if the patterns reported in our study are robust enough when considering the presence or absence of secondary resources in the snake diet. We described two matrices of interactions: (1) a matrix in which only main resources were considered; (2) and a matrix in which both main and secondary resources were considered. We defined if a resource is main or secondary according to information about snake diet preferences available in Martins and Oliveira (1998). Then, we calculate the nestedness and modularity values in the presence and absence of secondary resources. The nestedness values of the two networks were compared with a null model generated with 5,000 random removals of food resources from each of the analysed networks. Finally, we calculated whether there was a significant difference between the nestedness of the network in the presence and absence of secondary resources.

Because taxonomic resolution might influence the detection of patterns in the network (Rezende et al. 2009), we performed another sensitivity analysis to check if the type of resource categorization could affect the network patterns. Thus, we described two other matrices of interaction with different degrees in the resources taxonomic resolution: less specific network (Supplementary material Appendix 1 Figure A2 and Appendix 3 Table A1) and more specific network (Supplementary material Appendix 1 Figure A3 and Appendix 4 Table A1).

\section{Results}

\section{Network structure}

We recorded 163 interactions between 62 snake species and 26 food resources (Figure 1 and Supplementary material Appendix 1 Figure A6) that were heterogeneously distributed among snake species, where most of them had few interactions (56.45\% snake species interacted with one or two resource categories) and few species had many interactions $(6.45 \%$ interacted with more than five resources; Supplementary material Appendix 1 Figure A1). The network structure show moderate connectance $(\mathrm{C}=0.101)$ (Table 1$)$, indicating that, from the variety of food items consumed by snakes, the species analysed use, on average, 2-3 resources. The snake-resource network also show significant nestedness $(\mathrm{N}=33.14, \mathrm{p}<0.01)$, indicating that $1 / 3$ of the interactions of the less connected species represent a subset of the interactions of the most connected species. Finally, the network also show significant modularity $(\mathrm{M}=0.51, \mathrm{p}=0.03)$, indicating that the number of interactions within each modules is $51 \%$ larger than what is expected for a network with the same number of modules, the same number of interactions per species, but with random interactions between species.

Some snake species showed extreme specialisation, such as Dipsasspp., which feed exclusively on mollusks, and Drepanoides anomalus that rely upon eggs of squamate reptiles. Other species, such as Atractus spp., although specialist in the consumption of earthworms, may also feed on insects. Similarly, Micrurus surinamensis primarily consume fish but secondarily consume lizards and snakes. On the other hand, we found very generalist species, such asBoa constrictor and Epicrates cenchria, which interacted with six resource types, Corallus hortulanus, which interacted with eight resources and Eunectes murinus, the largest species of the network, which interacted with 11 resources. Among the food resources consumed by many snake species were lizards (24\% of all interactions), anurans (16\%), and small mammals (9\%), comprising rodents and marsupials. Among the least consumed resources were large mammals (such as cervids), turtles and alligators, only consumed byEunectes murinus, onychophorans only consumed by Micrurus hemprichii , gymnophiona only consumed by M. lemniscatus, and salamanders, which were only consumed by Chironius fuscus (Supplementary material Appendix 2 Table A1).

To assess whether there was a difference in network structure based only on the presence of primary resources in the snake diet, we removed all non-primary resources and reanalysed the network. Even after removing the secondary resources, network average degree and connectance remained within the same range values (Table 1). The results also indicated that there was no significant difference between the network nestedness values 
with and without the presence of secondary resources $(\mathrm{p}=0.147)$. Even after the removal of non-primary items, the network remained significantly nested $(\mathrm{N}=29.46, \mathrm{p}<0.01)$. In contrast, the modular structure was nonsignificant after removal of non-primary resources $(M=0.47, p=0.44)$. Similarity, to check if the type of food resource categorization could affect the network patterns, we used the same metrics to analyse the more specific and the less specific networks. Our results hold even with different levels of detail on resource description and all networks remained significantly nested and modular (Supplementary material Appendix 1 Table A2).

Our results supported the prediction that there is a positive association between the number of resources consumed and average body size (slope $=1.41, \mathrm{R}^{2}=0.46, \mathrm{p}<0.01$, Figure 2), indicating that in general the largest species of snakes showed a greater number of food interactions. Exceptions to this pattern were Corallus caninus $(\mathrm{k}=3)$ and Lachesis muta $(\mathrm{k}=1)$, both specialists in the consumption of mammals. Among the seven largest snake species, five of them (Eunectes murinus, Boa constrictor, Epicrates cenchria, Corallus hortulanus, and Corallus caninus ) belong to the family Boidae. Thus, this family is over-represented among the set of heavier snakes in the network and our analysis may be biased by the confounding factors generated by all other traits shared by boid species. To circumvent this problem we explored if the correlation between average body mass and degree holds within speciose snake families. We performed correlation analyses between degree and average body mass for species of the family Colubridae and for those of the family Dipsadidae, the two largest snake families in the network. The results indicated that a positive correlation between average body mass and the number of resources consumed hold even for non-boid snakes and partially controlling for phylogenetic effects, there was (see Supplementary material Appendix 1 Figure A4 and A5).

\section{The role of different species of snakes in network structure}

The correlation between degree and average body mass suggest nestedness was driven by body size. In fact, there is a positive correlation between delta nestedness and average body mass, indicating that the largest snakes have a greater contribution to nestedness (slope $=0.4, \mathrm{R}^{2}=0.37, \mathrm{p}<0.01$, Figure 3 ). We performed an removal analysis to further explore this pattern. We removed species with more outlier values of average body mass and recalculated the nestedness value. They were the seven largest snake species in the network (Eunectes murinus, Boa constrictor, Lachesis muta, Epicrates cenchria, Corallus hortulanus, Corallus caninus andSpilotes pullatus ). If the largest snakes are key components contributing to nestedness, we expected that nestedness after removal of these species would be smaller than those generated by a null model in which we randomly remove any seven species from the network and recalculate the nestedness. Our results supported this prediction, indicating that the nestedness values were smaller after the removal of the seven largest snake species $(\mathrm{N}=30.23, \mathrm{p}<0.01, \mathrm{n}=1,000$ simulations of species removal).

\section{The relationship between modularity and snake lifestyle}

The network also presented a modular structure, in which the consumption of different resources divided the network into six different food modules (Table 1 and Supplementary material Appendix 1 Table A3). We expected that if the snake lifestyle was related to the formation of food modules, the distribution of lifestyles in the different modules would not be random. In fact, modules were associated with particular lifestyles, as indicated by significant or marginally significant probability values present in all modules but module 3 (Table 2). Module 3, was composed of species with the greatest variety in diet and lifestyle, such as boid snakes. Modules with more specific combinations of lifestyle and diet showed all significant or marginally significant probability values (Figure 4). Moreover, the formation of groupings based on diet and lifestyle occurred by species that specialise in the consumption of certain food resources, probably associated with their lifestyle. For example, the smallest module (number 5) was formed by only two arboreal species of the genus Dipsas that feed exclusively on mollusks; another module grouped species of terrestrial habits, such as Drepanoides anomalus, Drymoluber dichrous, andMastigodryas boddaerti, which feed on squamate eggs, whereas another module grouped fossorial species, such as Atractus spp. that are specialists in preying upon earthworms. On the other hand, the remaining modules grouped species with varied lifestyles and generalist diets. 
As a consequence of the association between lifestyles and modular structure, modules often show dissimilar combinations of lifestyles. Dissimilarity values ranged from 0.33 to 1 (Table 2). The most dissimular module (Module 0) was composed exclusively of snakes with the fossorial lifestyle, such as the Atractus species, specialists in the consumption of earthworms. The most similar modules were modules 1 and 3 , which as well as module 2 have the largest variety of lifestyles and food resources consumed by the snakes (Supplementary material Appendix 1 Table A3).

\section{Discussion}

Our results indicated that the network of interactions between snakes and their resources in a species-rich Amazonian community presented a combination of both nested and modular structure. Nestedness was related to average body mass of snakes, in which boid snakes connect food modules in the trophic network. The modular pattern, in turn, is associated to the different snake lifestyles, in which snakes that share similar habits usually consume similar resources available in their shared microhabitats.

The observed connectance of the network indicated that, given the variety of resources available in the environment, snake species consumed only a subset of these resources. This result suggests that most food resources may not be accessible to most species, suggesting forbidden interactions (Olesen et al. 2010) associated with possible restrictions related to lifestyle (see Savitzky 1983), as well as body size (Sinclair et al 2003, Woodward et al. 2005, Stouffer et al. 2011). For instance, arboreal snakes have morphological adaptations, such as a slender body and long tail, which may represent limitations to the consumption of large prey such as mammals (Martins et al. 2001, Alencar et al. 2013, Alencar et al. 2017). The analysis of network structure revaled that the patterns of resource use by different species lead, at the community level, to nestedness and modularity. Our results contrast with some studies on antagonistic networks that indicate opposite trends between nestedness and modularity (Thébault and Fontaine 2010, Pires and Guimarães 2012). Having said that, other studies simultaneously show levels of nestedness and modularity (Bellay et al. 2011, Flores et al. 2013, Pinheiro et al. 2019). The emergence of these combined network patterns is possible due to the low connectivity of the network (Lewinsohn et al. 2006, Fortuna et al. 2010) and resource heterogeneity (Pinheiro et al. 2019) in Amazonian forests.

Several processes may explain the nested patterns, such as variations in species abundances (Lewinsohn et al. 2006). One of the explanations for the nested pattern found in our study was the large size variation among species present in the network. The variation in snake body mass has led to a trophic hierarchy in which larger predators prey upon more resource items than smaller predators. This hierarchy was detected in several predator-prey interaction networks found in nature (Woodward et al. 2005, Smith and Mills 2008, Arim et al. 2010). This pattern indicates that predators have the potential to add resources sequentially as they increase in size, although this increment of larger resources may lead to the rejection of smaller, less nutritious or difficult to handle resources (Mittelbach 1981, Arnold 1993, Arim et al. 2010, Woodward et al. 2010). In addition to body size, skull morphology is also an important feature associated with diet and snake lifestyle (Pough and Groves 1983, Savitzky 1983, Klaczko et al. 2016). The larger the head of a snake, the greater the prey consumed (King 2002). Thus, future research that investigates emerging patterns arising from the association of both body size and skull morphology with the structure of trophic interaction networks could predict the processes, at the community-level, involved in the relationships between snakes and their food resources.

When analysing the contribution of each species to nestedness, we found that average body mass has a phylogenetic signal, with large species concentrated in a few clades. After removing larger species, mostly boids, the nestedness value decreases $8.78 \%$ although it still remains significant. The maintance of nestedness after the removal of large snakes might be a consequence of the number of resource - body mass association holds for smaller snake species, such colubrid and dipsadid snakes. Boids are efficient constrictors with generalist diets, that occupy diverse microhabitats, which allow them to consume a wide variety of food resources (Pizzato et al. 2009, Henderson and Pauers 2012). This combination of features may simultenouly explain why (i) boids act as hubs (species with many interactions) in the analysed network, and (ii) the decrease in nestedness when boids are removed from the network. Large predators, such as sharks, killer 
whales, lions, and birds of prey, often prey on diverse array of species (Sinclair et al. 2003), potentially connecting modules in networks (e.g., Rezende et al. 2009). Future research could test whether the presence of such large predators can also promote nestedness on predator-prey interaction networks.

The modular structure in ecological networks may be associated to factors such as the degree of specialisation among interacting species (Prado and Lewinsohn 2004, Lewinsohn et al. 2006), habitat heterogeneity (Pimm and Lawton 1980), the phylogenetic relationship between species (Lewinsohn et al. 2006), the convergence in a set of species traits (Olesen et al. 2007) or by a combination of factors (Donatti et al. 2011). We found that the consumption of specific resources is associated with more peculiar lifestyles. For instance, morphological adaptations to fossorial habit (e.g. less cranial mobility) hinder the consumption of prey larger than the snake's head size (Greene 1983, Savitzky 1983, Martins and Oliveira 1993). Accordingly, arboreal habits impose physical limitations on snake morphology and may restrict the consumption of larger prey, such as small mammals, favoring a diet based on lizards and/or frogs (Lillywhite and Henderson 1993, Martins et al. 2001, Martins et al. 2002, Alencar et al. 2013). Thus, we suggest that the modularity of the network we studied has emerged from the relationship between the lifestyles of snakes and the consumption of resources restricted to the habitats used by the species.

To sum up, we integrate network structure analyses with species removal simulations to evaluate the role of different snake species in the structure of a rich Amazonian snake community, and the mechanisms underlying the patterns found. We encourage future studies to focus on understanding how community phylogenetic diversity may be associated with the modular structure (Rezende et al. 2009), as well as how the combination of traits associated with predator diet (e.g. its correlation with body size and skull shape) may contribute to the nested pattern and if geographic variation (environment type) can modify network structure (Pimm and Lawton 1980, Kortsch et al. 2019). This study points to the joint importance of the evolutionary history of lineages, body size, and their interacting resources to determine the structure, at the community scale, of the interactions between consumers and their resources.

\section{Data Accessibility Statement}

We declare that all data supporting the conclusions of this study (dataset, and supplementary information) are uploaded in Dryad ( DOI https://doi.org/10.5061/dryad.f1vhhmgvt.) and will be available by the time the paper is accepted.

\section{References}

Abram, P. A. 1987. On classifying interactions between populations. - Oecologia 73: 272-281.

Alencar, L. R. V. et al. 2013. The Evolution of Diet and Microhabitat Use in Pseudoboine Snakes. - South Am. J. Herpetol. 8: 60-66.

Alencar, L. R. V. et al. 2017. Arboreality constrains morphological evolution but not species diversification in vipers. - Proc. Royal Soc. B 284: 20171775.

Almeida-Neto, M. et al. 2008. A consistent metric for nestedness analysis in ecological systems: reconciling concept and measurement. - Oikos 117: 1227-1239.

Araújo, M. S. et al. 2008. Network analysis reveals contrasting effects of intraspecific competition on individual vs. population diets. - Ecology 89: 1981-1993.

Arim, M. et al. 2010. Food web structure and body size: trophic position and resource

Acquisition. - Oikos 119: 147-153.

Arnold, S. J. 1993. Foraging theory and prey size - predator-size relations in snakes. - In Seigel, R. A. and Collins, J. T. (eds.), Snakes: Ecology and Behavior. McGraw-Hill, New York, pp. 87-115.

Barber, M. J. 2007. Modularity and community detection in bipartite networks. - Phys. Rev. E 76: 066102.

Bascompte, J. et al. 2003. The nested assembly of plant-animal mutualistic networks. - PNAS 100: 9383-9387. 
Bascompte, J. and Jordano, P. 2013. Mutualistic Networks. - Princeton: Princeton University Press.

Bellay, S. et al. 2011. A host-endoparasite network of Neotropical marine fish: are there organizational patterns? - Parasitology 138: 1945-1952.

Bellini, G. P. et al. 2015. Temperate Snake Community in South America: Is Diet Determined by Phylogeny or Ecology? - Plos One 10: 1-15.

Cohen, J. E. 1977. Food Webs and Niche Space. Princeton University Press, Princeton, New Jersey, USA.

Colston, T. J. et al. 2010. Snake diets and the deep history hypothesis. - Biol. J. Linn. Soc. 101: 476-486.

Covich, A. P. and McDowell, W. H. 1996. The stream community. - In Reagan D. P. and Waide, R. B. (eds), The food web of a tropical rainforest. University of Chicago Press, Chicago, Illinois, pp. 433-459.

Donatti, C. I. et al. 2011. Analysis of a hyper-diverse seed dispersal network: modularity and underlying mechanisms. - Ecol. Letters 14: 773-781.

Feldman, A. et al. 2016. Body sizes and diversification rates of lizards, snakes, amphisbaenians and the tuatara. - Global Ecol. Biogeogr. 25: 187-197.

Flores, C. O. et al. 2013. Multi-scale structure and geographic drivers of cross-infection within marine bacteria and phages. - ISME J. 7: 520-532.

Fortuna, M. A. et al. 2010. Nestedness versus modularity in ecological networks: two sides of the same coin? - J. Anim. Ecol. 79: 811-817.

Greene, H. W. 1983. Dietary correlates of the origin and radiation of snakes. - Amer. Zool. 23: 431-441.

Guimarães, P. R. 2020. The structure of ecological networks across levels of organization. - Annual Review of Ecology, Evolution, and Systematics (in press).

Guimera, R. and Amaral, L. A. 2005. Functional cartography of complex metabolic networks. Nature 433: 895-900.

Henderson, R. W. and Pauers, M. J. 2012. On the diets of neotropical treeboas (Squamata: Boidae: Corallus ). - South Am. J. Herpetol. 7: 172-180.

Ings, T. C. et al. 2009. Ecological networks - beyond food webs. - J. Anim. Ecol. 78: 253- 269.

King, R. B. 2002. Predicted and observed maximum prey size-snake size allometry. - Funct. Ecol. 16: 766-772.

Klaczko, J. et al. 2016. Are Diet Preferences Associated to Skulls Shape Diversification in Xenodontine Snakes? - PLoS One 11: 1-12.

Kortsch, S. et al. 2019. Food-web structure varies along environmental gradients in a high-latitude marine ecosystem. - Ecography 42: 295-308.

Krause, A. E. et al. 2003. Compartments revealed in food-web structure. - Nature 426: 282-285.

Krishna, A. et al. 2008. A neutral-niche theory of nestedness in mutualistic networks. - Oikos 117: 16091618 .

Lewinsohn, T. M. et al. 2006. Structure in plant-animal interaction assemblages. - Oikos 113: 174-184.

Lillywhite, H. B. and Henderson, R. W. 1993. Behavioral and functional ecology of arboreal snakes. - In: Seigel, R. A. and Collins, J. T. (eds.), Snakes: ecology and behavior. New York: McGraw-Hill, pp. 1-48.

Marquitti, F. M. D. et al. 2014. MODULAR: Software for the autonomous computation of modularity in large network sets. - Ecography 37: 221-224. 
Martins, M. and Oliveira, M. E. 1993. The snakes of the genus Atractus Wagler (Reptilia: Squamata: Colubridae) from the Manaus region, central Amazonia, Brazil. - Zool. Meded. 67: 21-40.

Martins, M. and Oliveira, M. E. 1998. Natural history of snakes in forests of the manaus region, Central Amazonia, Brazil. - Herpetol. Nat. History 6: 78-150.

Martins, M. et al. 2001. Diversity and evolution of macrohabitat use, body size and morphology in a monophyletic group of Neotropical pitvipers (Bothrops). - J. Zool. 254: 529-538.

Martins, M. et al. 2002. Ecological and phylogenetic correlates of feeding habits in Neotropical pitvipers (Genus Bothrops ). - In: Schuett, G. W. et al. (eds.), Biology of the vipers. Eagle Mountain Publishing, Eagle Mountain, pp. 307-328.

Mittelbach, G. G. 1981. Foraging efficiency and body size: a study of optimal diet and habitat use by Bluegills. - Ecology 62: 1370-1386.

Oksanen, J. et al. 2018. vegan: Community Ecology Package. R package version 2.5-2. https://CRAN.Rproject.org $/$ package $=$ vegan .

Olesen, J. M. et al. 2007. The modularity of pollination networks. - PNAS. 104: 19891-19896.

Olesen, J. M. et al. 2010. Missing and forbidden links in mutualistic networks. - Proc. Royal Soc. B 278: 725-732.

Pimm, S. L. and Lawton, J. H. 1980. Are food webs compartmented? - J. Anim. Ecol. 49, 879-898.

Pinheiro, R. B. P. et al. 2019. A new model explaining the origin of different topologies in interaction networks. - Ecology 100: e02796.

Pires, M. M. and Guimaraes, P. R. 2012. Interaction intimacy organizes networks of antagonistic interactions in different ways. - J. R. Soc. Interface 10: 20120649.

Pizzatto, L. et al. 2009. Food habits of Brazilian boid snakes: overview and new data, with special reference to Corallus hortulanus . - Amphibia-Reptilia 30: 533-544.

Pough, H. and Groves, J. D. 1983. Specializations of the Body Form and Food Habits of Snakes. - Am. Zool. 23: 443-454.

Prado, P. I. and Lewinsohn, T. M. 2004. Compartments in insect-plant associations and their consequences for community structure. - J. Anim. Ecol. 74: 1168-1178.

R Core Team. 2018. R: A language and environment for statistical computing. R Foundation for Statistical Computing, Vienna, Austria. URL https://www.R-project.org/.

Rezende, E. L. et al. 2009. Compartments in a marine food web associated with phylogeny, body mass, and habitat structure. - Ecol. Lett. 12: 779-788.

Savitzky, A. H. 1983. Coadapted Character Complexes Among Snakes: Fossoriality, Piscivory, and Durophagy. - Am. Zool. 23: 397-409.

Schmitz, O. J. and Beckerman, A. P. 2007. Food Webs. Encyclopedia of Life Sciences, John Wiley \& Sons. Schoener, T. W. 1968. The Anolis Lizards of Bimini: Resource Partitioning in a Complex Fauna. - Ecology 49: 704-726.

Shine, R. and Bonnet, X. 2000. Snakes: a new 'model organism' in ecological research? - TREE 15: 221-222.

Sinclair, A. R. E. et al. 2003. Patterns of predation in a diverse predator-prey system. - Nature 425: 288-290.

Smith, N. O. and Mills, M. G. L. 2008. Predator-prey size relationships in an African large-mammal food web. J. Anim. Ecol. 77: 173-183. 
Stouffer, D. B. et al. 2011. The role of body mass in diet contiguity and food-web structure. - J. Anim. Ecol. 80: 632-639.

Thebault, E. and Fontaine, C. 2010. Stability of Ecological Communities and the Architecture of Mutualistic and Trophic Networks. - Science 329: 853-856.

Thompson, J. N. 2005. The Geographic Mosaic of Coevolution. University of Chicago Press, Chicago.

Woodward, G. et al. 2005. Body size in ecological networks. - TREE 20: 402-409.

Woodward, G. et al. 2010. Individual-based foodwebs: Species identity, body size and sampling effects. Adv. Ecol. Res. 43: 211-266.

\section{Captions of the main text figures:}

Figure 1: Network describing the interactions (lines) between species of Amazonian snakes (circles) and their food resources (triangle).

Figure 2: The number of categories of food resources consumed by different snake species is positively associated with the snake average body mass (slope $=1.41, \mathrm{R} 2=0.46, \mathrm{p}<0.01$ ) in a network of interactions between Amazonian snakes and their resources. Each point represents a species and the colors represent the different snake families. To avoid overlap, some points have been slightly offset from their original position on the x-axis.

Figure 3: In simulations of species removal, changes in the level of nestedness are positively associated with the snake average body mass of the removed species ( slope $=0.40, \mathrm{R} 2=0.37, \mathrm{p}<0.01$ ). Note the stronger, negative changes on nestedness are associated with Boidae snakes (red).

Figure 4: The probability of the observed number of species of a given lifestyle in a given module of being reproduced randomly. Red color indicates higher frequency, with a 95\% confidence interval.

Table 1: Relationship of the network structure analysis of interactions between Amazonian snakes and their food resources. $\mathrm{S}=$ snake species richness in the network; $\mathrm{R}=$ food resources richness (Note that with the removal of species from the network occurs the loss of interactions, which may reduce the number of resources); $\mathrm{SD}=$ average degree; $\mathrm{C}=$ connectivity; $\mathrm{N}=$ nestedness; Nrel = relative nestedness; $\mathrm{M}=$ modularity; Mrel $=$ relative modularity; $\mathrm{nM}=$ number of food modules.

\begin{tabular}{llllllllllll}
\hline Web & S & $\mathbf{R}$ & SD & $\mathbf{C}$ & $\mathbf{N}$ & $\mathbf{p ~ N}$ & Nrel & $\mathbf{M}$ & $\mathbf{p ~ M}$ & $\mathbf{n M}$ & Mrel \\
\hline Complete web & 62 & 26 & 2.62 & 0.10 & 33.14 & $\mathrm{p}<0.01$ & 0.94 & 0.51 & $\mathrm{p}=0.03$ & 6 & 0.11 \\
Without secondary resources & 62 & 24 & 2.34 & 0.09 & 29.46 & $\mathrm{p}<0.01$ & 0.93 & 0.48 & $\mathrm{p}=0.44$ & 7 & 0.03 \\
Without the 7 largest species & 55 & 18 & 2.23 & 0.12 & 30.23 & $\mathrm{p}<0.01$ & 0.69 & 0.53 & $\mathrm{p}=0.06$ & 7 & 0.12 \\
Without species of Boidae & 57 & 19 & 2.26 & 0.12 & 30.42 & $\mathrm{p}<0.01$ & 0.74 & 0.53 & $\mathrm{p}=0.01$ & 7 & 0.13 \\
\hline
\end{tabular}

Table 2: Comparison between the real interaction matrix, the dissimilarity matrix, and the likelihood matrix of the lifestyle by food module of the network of interactions between Amazonian snakes and their food resources. Lines represent the six (0-5) food modules and columns represent the lifestyles of the snakes. Asterisks represent significant or marginally significant values of probability.

\section{Real matrix}

\begin{tabular}{lllll}
\hline Modules & Aquatic & Arboreal & Fossorial & Semi-arboreal \\
$\mathbf{0}$ & 0 & 0 & 10 & 0 \\
$\mathbf{1}$ & 1 & 9 & 0 & 0 \\
$\mathbf{2}$ & 2 & 0 & 3 & 1 \\
$\mathbf{3}$ & 1 & 5 & 0 & 1 \\
$\mathbf{4}$ & 0 & 0 & 0 & 0
\end{tabular}




$\begin{array}{lllll}\mathbf{5} & 0 & 2 & 0 & 0 \\ \text { n species } & 4 & 16 & 13 & 2 \\ \text { Dissimilarity matrix } & \text { Dissimilarity matrix } & \text { Dissimilarity matrix } & \text { Dissimilarity matrix } & \text { Dissimilarity mat } \\ \text { Modules } & \mathbf{0} & \mathbf{1} & \mathbf{2} & \mathbf{3} \\ \mathbf{0} & 0 & 1 & 0.714 & 1 \\ \mathbf{1} & 1 & 0 & 0.706 & 0.333 \\ \mathbf{2} & 0.714 & 0.706 & 0 & 0.667 \\ \mathbf{3} & 1 & 0.333 & 0.667 & 0 \\ \mathbf{4} & 1 & 0.769 & 0.714 & 0.625 \\ \mathbf{5} & 1 & 0.840 & 1 & 0.733 \\ \text { Probability matrix } & \text { Probability matrix } & \text { Probability matrix } & \text { Probability matrix } & \text { Probability matri } \\ \text { Modules } & \text { Aquatic } & \text { Arboreal } & \text { Fossorial } & \text { Semi-arboreal } \\ \mathbf{0} & 1 & 1 & 0 * & 1 \\ \mathbf{1} & 0.839 & 0.06^{*} & 1 & 1 \\ \mathbf{2} & 0.152 & 1 & 0.41 & 0.303 \\ \mathbf{3} & 0.647 & 0.197 & 1 & 0.386 \\ \mathbf{4} & 1 & 1 & 1 & 1 \\ \mathbf{5} & 1 & 0.078^{*} & 1 & 1\end{array}$

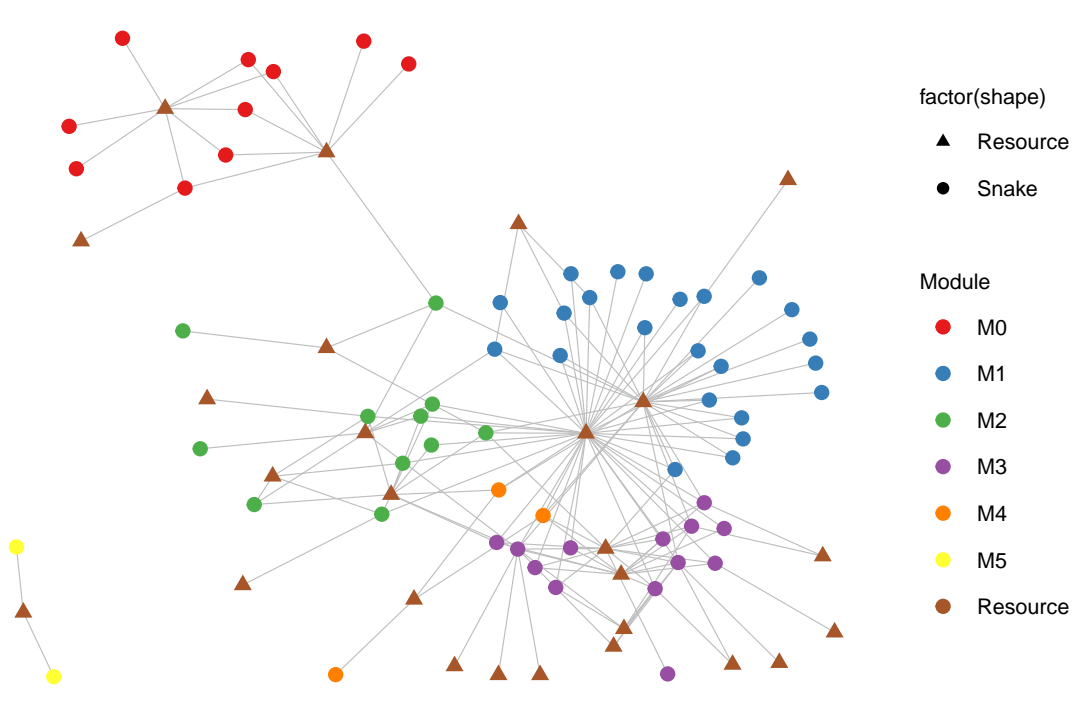




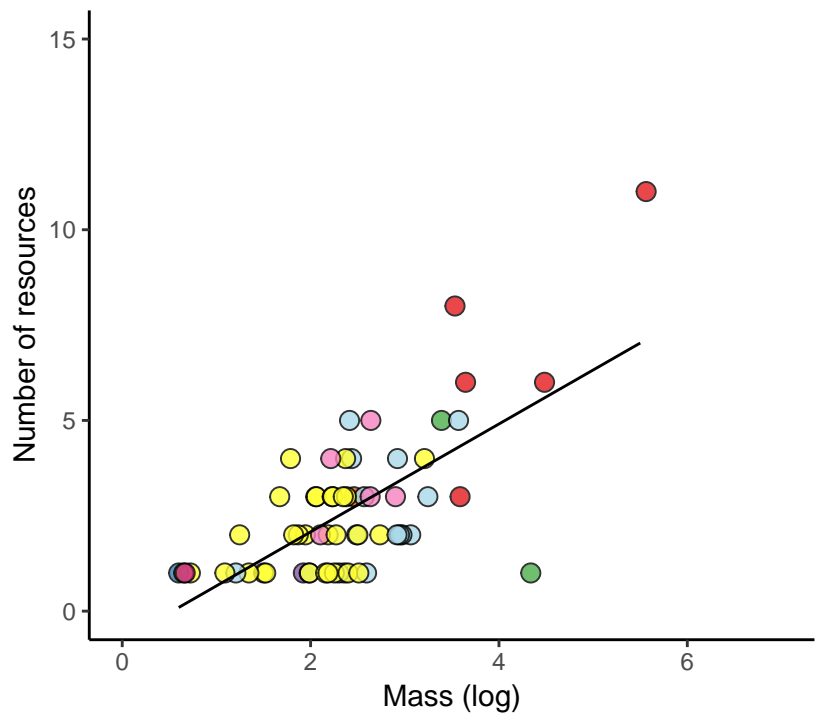

Family

$\bigcirc$ Aniliidae

Anomalepididae

- Boidae

C Colubridae

○ Dipsadidae

- Elapidae

Leptotyphlopidae

Typhlopidae

V Viperidae

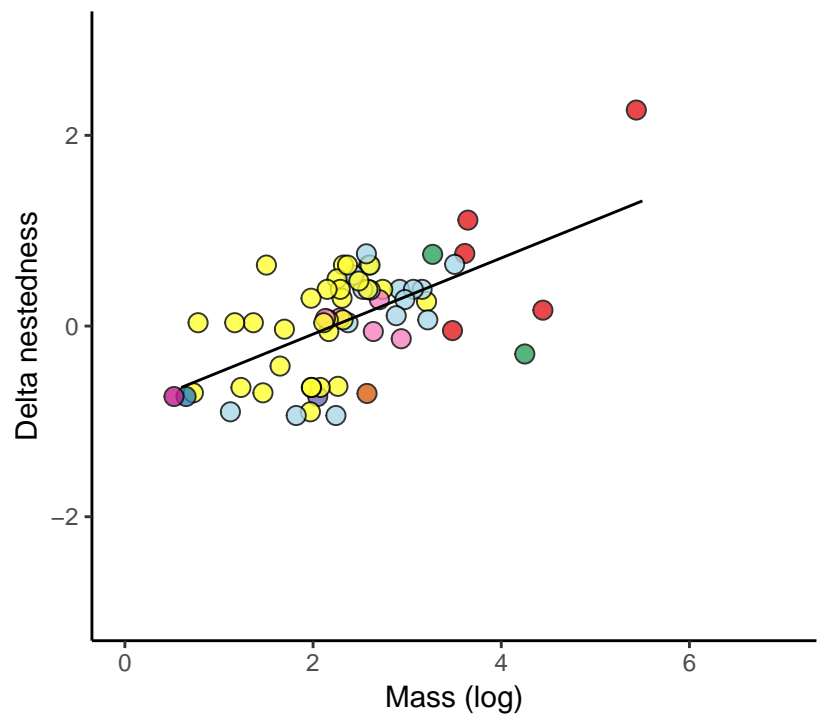

Family

- Aniliidae

- Anomalepididae

- Boidae

O Colubridae

O Dipsadidae

O Elapidae

- Leptotyphlopidae

O Typhlopidae

○ Viperidae 


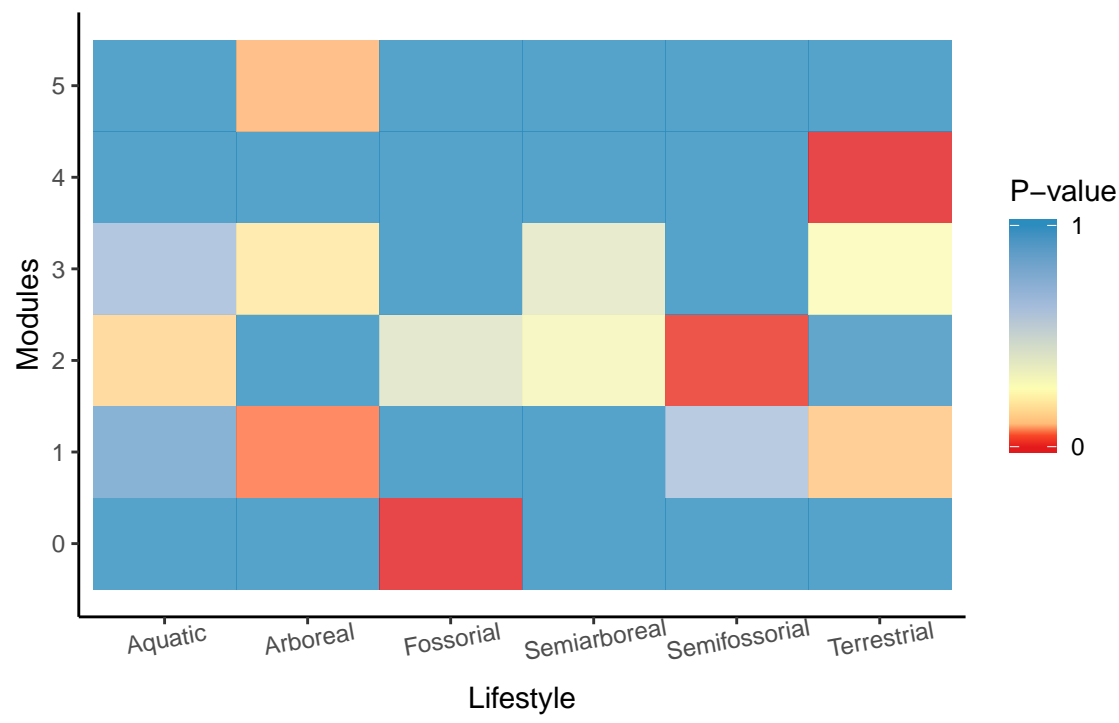

